\section{Betting on our future}

S cientific 'grantsmanship' can be viewed as analyzing and supporting a risky idea (a 'bet') and winning grants to fund the exploration of that idea. However, winning does not come easily, because although ideas are plentiful, funds are finite. The current system of peer review is perceived as the fairest and most effective way to maximize limited resources, but is it? Unexpectedly, a recent US government public relations fiasco could rekindle a decade-old, and highly radical, idea to boost the efficiency of directing scientific research.

Just a day after it was announced in July 2003, the Policy Analysis Market (PAM), went down in a ball of fire. PAM was to be a 'futures market' on political developments related to the Middle East. Unforeseen by the planners at the US Defense Advanced Research Project Agency (DARPA, the same agency that originally funded and developed the Internet), the proposed 'betting' on events such as political upheavals and assassinations incited massive public outrage. However, the initiative is based on an intellectually fascinating set of logic.

Inspired by the work of economist Robin Hanson from George Mason University, PAM would have provided a market in which people could speculate on the likelihood of political, civil and military events in the Middle East. The logic behind using a futures market stems from the hypothesis that future events can be more accurately predicted by collective, rather than individual, knowledge. The Iowa Electronic Markets (http://www.biz.uiowa.edu/iem/index.html) trades in the fortunes of political candidates, and it has an excellent record predicting the results of US elections, compared with opinion polls and expert analysts. Similarly, it was hoped that PAM would better predict the volatile Middle East by enticing people who have pertinent information to profit from their knowledge. Not surprisingly, the project has attracted skeptics (for media coverage and discussion, see http://hanson.gmu.edu/ myPAMpress.html).

Could such an idea be relevant to scientific research? Using a similar framework, Robin Hanson explored the concept of an idea futures' market a decade ago ("Could Gambling Save Science"; http://hanson.gmu.edu/gamble.html). Hanson proposed a market system for scientific research that would help a question to "converge as fast as possible to the right answer." The proposed market is traded like a futures exchange, except a 'trade contract' would be whether a vaccine for SARS will be developed by April 2005 rather than the price of peanuts in October 2004. An individual would bet on the likelihood of a scientific outcome based on his or her own information. Extracting the collective probabilistic forecast from this market could shape the direction of research. Thus, to gain an advantage on the market, scientists could focus their research on a particular contract in the market. Funding agencies could also choose to distribute their funds by sponsoring a trade contract, rather than a research group.

Because individuals speculating on the likelihood of a scientific result make a personal investment, the market provides a strong incentive for thoughtfulness and honesty. This system also promotes the 'right' scientific idea rather than the trendy one, because any scientific question could be traded, with rewards for the right answer. But perhaps the most important advantage of the futures market is the provision of a medium for cooperative knowledge to drive scientific research at a quicker pace. One might argue that scientific publications and meetings serve this exact purpose. However, that would erroneously assume encyclopedic knowledge of available information, without taking into account important data that are not publicized at meetings or are too preliminary for publication.

Of course, such an extraordinary idea raises many questions. How could funding agencies justify sponsoring a contract that will be awarded, at least partially, to speculators of the market? Can the rich and powerful skew the market? Will this system stifle creativity? Hanson attempted to address some of these concerns in his article. The futures market idea does not propose a sweeping overnight change from the current system. Instead, incremental steps backed by positive results would be necessary to convince the community. Interestingly, an ongoing project based on the original idea futures, the Foresight Exchange (http://www.ideosphere.com/), allows one to propose an idea contract and trade on those ideas, including scientific experiments.

Perhaps the scientific community is not yet ready for a true idea futures market. The participation of the general public dilutes the pool of expert knowledge that would shape the speculation, and a quest for knowledge that is driven by profit is considered somewhat 'unsavory'. However, a market stripped of those complications is still potentially useful if enough scientists are willing to contribute in the interest of advancing science (of course, one of the fundamentals of the Hanson's model is the monetarily driven incentive, which a 'stripped-down' market would lack). All scientists have personal insights and opinions on current scientific questions. However, not all scientists have an opportunity to share their views. A futures market would offer a venue where the 'right' direction could be synthesized from collective knowledge. Concurrently, a futures market also rewards those who show the foresight to bet against flawed trends.

Effective distribution of resources for scientific research is a paramount concern for both scientists and funding agencies. A system that uses the community's collective wisdom to determine the level of interest and the 'correctness' of an idea makes a compelling case for further discussion. How long are the odds that injecting explicit rewards for expressed risks in a futures market would not benefit us all? 